This item was submitted to Loughborough's Research Repository by the author.

Items in Figshare are protected by copyright, with all rights reserved, unless otherwise indicated.

\title{
Modelling water intrusion and oxygen diffusion in a reconstructed microporous layer of PEM fuel cells
}

\section{PLEASE CITE THE PUBLISHED VERSION}

http://dx.doi.org/10.1016/j.ijhydene.2014.08.027

\section{PUBLISHER}

(C) Elsevier

VERSION

AM (Accepted Manuscript)

\section{PUBLISHER STATEMENT}

This work is made available according to the conditions of the Creative Commons Attribution-NonCommercialNoDerivatives 4.0 International (CC BY-NC-ND 4.0) licence. Full details of this licence are available at: https://creativecommons.org/licenses/by-nc-nd/4.0/

\section{LICENCE}

CC BY-NC-ND 4.0

\section{REPOSITORY RECORD}

Zhang, Xiaoxian, Yuan Gao, Hossein Ostadi, Kyle Jiang, and Rui Chen. 2015. "Modelling Water Intrusion and Oxygen Diffusion in a Reconstructed Microporous Layer of PEM Fuel Cells". figshare. https://hdl.handle.net/2134/16565. 


\title{
Accepted by International Journal of Hydrogen Energy
}

\section{Modelling water intrusion and oxygen diffusion in a reconstructed microporous layer of PEM fuel cells}

\author{
Xiaoxian Zhang ${ }^{\mathrm{a}}$, Yuan Gao ${ }^{\mathrm{b}}$, Hossein Ostadi ${ }^{\mathrm{c}}$, Kyle Jiang ${ }^{\mathrm{d}}$ Rui Chen $^{\mathrm{e}}$
}

${ }^{\text {a }}$ School of Engineering, University of Liverpool, Brownlow Street, Liverpool, L69 3GQ UK.

e-mail: Xiaoxian.zhang@liverpool.ac.uk

${ }^{\mathrm{b}}$ Clean Energy Automotive Engineering Centre \& School of Automotive Studies, Tongji University, Shanghai 201804, China e-mail: yuangao@tongji.edu.cn

${ }^{\mathrm{c}}$ Department of Mechanical and Manufacturing Engineering, University of Birmingham, BirminghamB15 2TT, UK. e-mail: ostadi@contacs.bham.ac.uk

${ }^{\mathrm{d}}$ Department of Aeronautical and Automotive Engineering, Loughborough University, Leicestershire LE11 3TU, UK. e-mail: R.Chen@lboro.ac.uk

Corresponding author;

Dr. X. Zhang, School of Engineering, University of Liverpool, Brownlow Street, Liverpool L69 3GQ. UK

Tel: +44 1517945219

E-mail: xiaoxian.zhang@liverpool.ac.uk 


\begin{abstract}
The hydrophobic microporous layer (MPL) in PEM fuel cell improves water management but reduces oxygen transport. We investigate these conflict impacts using nanotomography and porescale modelling. The binary image of a MPL is acquired using FIB/SEM tomography. The water produced at the cathode is assumed to condense in the catalyst layer (CL), and then builds up a pressure before moving into the MPL. Water distribution in the MPL is calculated from its pore geometry, and oxygen transport through it is simulated using pore-scale models considering both bulk and Knudsen diffusions. The simulated oxygen concentration and flux at all voxels are volumetrically averaged to calculate the effective diffusion coefficients. For water flow, we found that when the MPL is too hydrophobic, water is unable to move through it and must find alternative exits. For oxygen diffusion, we found that the interaction of the bulk and Knudsen diffusions at pore scale creates an extra resistance after the volumetric average, and that the conventional dusty model substantially overestimates the effective diffusion coefficient.
\end{abstract}

Key words: PEM fuel cells; micro-porous layer; FIB/SEM tomography; pore-scale modeling; saturation; dusty model. 


\section{Introduction}

Water management is a crucial issue in polymer electrolyte membrane (PEM) fuel cell [1].To facilitate proton transport through the membrane, the hydrogen is normally humidified externally at the anode, whilst to avoid flooding, the water produced at the cathode needs to be moved out of the cell. Humidifying hydrogen at one side and removing water from the other side is self-defeating, and an improved design is desirable [2].

Recent studies found that adding a hydrophobic micro-porous layer (MPL) between the gas diffusion layer (GDL) and the catalyst layer (CL) at the cathode can improve water management [3, 4]. The hydrophobic MPL functions as a hydraulic barrier, forcing water condensed in the CL to build up a pressure before it can move into the MPL [5]. This increased pressure can drive the water moving backwards into the membrane so as to keep it hydrated [6]. Whilst such a MPL improves water management, it could reduce oxygen diffusion from the GDL to the CL. These conflicting impacts mean that an optimal design is necessary [7]. A essential parameter in MPL design is the impact of its saturation on water flow and oxygen diffusion [8]. Since the MPL is only a few microns thick, experimentally measuring these parameters is difficult [9].

Pore-scale modelling and tomography can be used in a combination to bridge this gap $[10,11]$. For example, using X-ray computed tomography or focused ion beam/scanning electron microscopy (FIB/SEM), one can visualise the interior structure of a porous material at resolutions as fine as a few nanometres [12-17]. There has been a surge over the past few years in use of pore-scale model and tomography to simulate various flow and transport processes in PEM fuel cells, including twophase flow [18], oxygen diffusion and reduction in cathode CL [19], and estimating effective transport parameters $[11,20]$. These have substantially improved our understanding of some fundamental processes occurring in the cell[17], which would remain unknown otherwise [21].

The hydrophobic MPL is capable of improving water management, but the underlying mechanisms are not fully understood and still a subject of debate [22]. The earlier theoretical 
models hypothesized that the water produced in the cathode condensed in the CL first, and it was then driven into the MPL by a capillary pressure gradient [23]. Since the MPL was assumed to be more hydrophobic than the CL, these models predicted that with an increase in MPL thickness, the water would gradually flood the part of MPL adjacent the CL. Recent experiments, however, have casted doubts over this hypothesis [24]. For example, the observation of Aoyama et al [24] showed that their MPL remained dry even after liquid water had accumulated at the CL-MPL interface, indicating that the water did not move into the MPL and must have found alternative exits. Since water movement in a medium is controlled by its pore geometry and hydrophobicity, understanding pore-scale processes is essential to help MPL design and has attracted increased interest, ranging from its structure characterization[25], process visualization [26], to numerical modelling [27]. At pore scale, Wargo et al.[20] measured the interior structure of a MPL using both X-ray and FIB/SEM tomographies, and then calculated its morphological parameters such as tortuosity and structural diffusivity.

PEM fuel cells work at low temperature and involve liquid water and gaseous reactants [28]. As a result, the MPL could be partly saturated; its saturation might change the ways of water flow and oxygen transport, and ultimately affects cell performance. Pant et al. [29] made an effort to experimentally measure the permeability of a dry MPL. But measuring the ability of a partly saturated MPL to simultaneously conduct liquid water and gaseous oxygen in opposite directions is difficult. Also, the average pore size in a MPL is approximately 150 nanometres [30], and the impact of Knudsen diffusion on oxygen transport in such pores could be significant. Furthermore, with saturation in a MPL increasing, the available space for oxygen to transport will reduce and the influence of Knudsen diffusion will become increasing profound. How a change in saturation affects water movement within an MPL and its consequent impact on oxygen diffusion is an important issue which is still poorly understood. For gas transport in media dominated by nanopores, the dusty model is usually used to estimate their effective diffusion coefficient, assuming that the 
interaction of the bulk and Knudsen diffusions at pore scale could be averaged out by a volumetric average[30, 31]. The reliability of this assumption, however, has not been well verified.

The purpose of this paper is to investigate pore-scale water flow and oxygen diffusion in the MPL under different saturations. The microstructure of a MPL was acquired using FIB/SEM tomography at resolution of 14 nanometres, and pore-scale models were developed to simulate water intrusion and oxygen diffusion in the structure under different saturations. In all simulations, the water was assumed to condense in the CL first, and it then built up a pressure to move into the MPL. The MPL is more hydrophobic, and the minimum pressure the water needs to build up in the CL to make it able to move through the MPL is called water-entry pressure. Water flow and its detailed distribution in the MPL were calculated from its pore sizes and pore connectedness. For a pressure built by water in the CL, we first determined if the pressure was high enough to drive water into the MPL, and if it was, we then modelled water intrusion and distribution in the MPL. Oxygen diffusion was restricted to the air phase, and was driven by a concentration gradient imposed in the GDL-CL direction; both bulk and Knudsen diffusions were considered. The simulated oxygen concentration and flux at all voxels were volumetrically averaged, and they were then used to calculate the effective diffusion coefficients under different saturations. We also tested the reliability of the dusty model by direct pore-scale simulations.

\section{Methodology}

\subsection{FIB/SEM imaging}

Details of the FIB/SEM technologies used for imaging microporous layer and catalyst layer of PEM fuel cells were given in previous work by us and others $[14,16]$. For completeness, however, we describe it there.

The principle of FIB/SEM is to mill away a slice of the sample as thin as $10 \sim 15 \mathrm{~nm}$ from the side wall of a trench using FIB, and then take an SEM image of the new surface as illustrated in Figure 1. Repeating the mill-image cycle produces a stack of 2D SEM images. The SEM images are 
then all aligned in reference to a fiducial mark milled prior to milling thin slices. The fiducial mark need to remain sharp and undistorted in each slice/image, and should be as tiny as possible to occupy only a small region of the SEM image at high magnifications. The current fiducial marking techniques invoke a large area which hampers imaging at high magnifications. These fiducial marks are also distorted during FIB imaging $[3,5]$. The problem can be resolved by milling a vertical slot close to the trench and position it out of the ion beam window as shown in Figure 1. Moreover, the bombardment of FIB tends to damage the underneath layer of the soft porous materials and deform the porous structure. To overcome such problems, a metal layer can be coated on the top of the sample and the sample is milled vertically. The metal layer also serves to suppressing curtaining effects. In this way, the damaged porous structures are removed, leaving the original structures intact for SEM imaging. Meanwhile, SEM images with a resolution of a few nanometres have enabled the technique.

The FIB/SEM nanotomography process (FEI Dual Beam Strata 235, Oregon, US: FEI Company) started with a deposition of $100 \mathrm{~nm}$ thick platinum layer on the top surface of MPL in order to protect the soft CL material from the ion bombardment and to reduce the re-deposition effect of FIB-induced decomposition of precursor gases. A slot as a fiducial mark was then milled for image alignment. The tiny slot is positioned out of the ion beam window to minimize the FIB damage effect. Next, each slice of the sample was milled-off using a $\mathrm{Ga}^{+}$ion beam at $30 \mathrm{kV}$ and 30 pA current with the FIB/SEM system. 100 slices with a total thickness of $1 \mu \mathrm{m}$ were removed and SEM images of the slices were taken. 2D images were then vertically stretched by a factor of 1.27 to compensate the SEM projection angle. Images are then finely aligned based on the fiducial mark with Lucas-Kanade algorithm [21] using Adobe Photoshop CS3 (www.adobe.com). Aligned images are thresholded to distinguish the solid network from the pore network through Otsu algorithm [22]. Finally, binary 2D images were assembled together to build a 3D binary image with 1 and 0 
representing solid and pore phases respectively using the CT software (www.skyscan.be). Figure 2 show an image acquired using this method.

\subsection{Pore diameter and water distribution}

We took one part of the image shown in Figure 2 for simulations due to computer-power limitation but ensured that it was big enough to be representative as discussed by Wargo et al. [32]. Figure $3 \mathrm{~A}$ shows the simulated image. Its porosity is approximately $31 \%$, and its sizes in all the three directions are 200 voxels.

To quantify pore sizes and pore connectedness, we assumed that the void space in the image is a packing of overlapped spherical air bubbles with different diameters. The diameter of a pore is defined as the diameter of the sphere that just occupies this pore. To determine the diameters of all the spheres, for each pore voxel shown in Figure 3A we firstly calculated its shortest distance to all solid walls. We then started from the pore voxel that is most distant from the solid walls by taking it as the centre of a sphere. The sphere was then expanded until it touched a solid wall; the void space within this expanded sphere was defined as a temporary pore, and its diameter was defined as the diameter of the sphere; all pore voxels inside the expanded sphere were assigned to the sphere diameter as their temporary identity labels. The final diameters of all pores were determined by repeating the above procedure to all pore voxels and progressively updating their temporary identity labels. For the overlapped spheres, the pore voxels within the overlapped zone took the diameter of the largest sphere as their identity labels. Figure 3A shows the pore-size distribution calculated using the above method.

Water movement and distribution in the MPL were assumed to be controlled by capillary pressure $p$ and connectedness of all the pores, which in turn depends on the pore radius $\delta$ in $p \propto \cos \theta \cdot \sigma / \delta$, where $\sigma$ is the air-water surface tension, and $\theta$ is the contact angle between MPL wall and the water-air interface. For a given contact angle, each pore is associated with a capillary pressure. To determine if the water in the CL was able to invade the MPL, we assumed that the 
bottom of the image shown in Figure 3A was in direct contact with a CL. Since the CL is less hydrophobic, the water in the CL needs to build up a pressure $p_{0}$ before it is able to move into the MPL. The minimum pressure the water needs to build up in order to move through the MPL is called water-entry pressure; it depends on hydrophobicity and pore geometry of the MPL.

The MPL is hydrophobic and its water contact angle is larger than $90^{\circ}$. Therefore, the large pores are less resistant to water flow and will be invaded first. To calculate water invasion under pressure $p$ built by water within the CL, we started from the pore voxels in direct contact with the CL to first check if the water could invade. Water would invade the pore voxels whose associated capillary pressures were less than $p$. If the outcome of this calculation resulted in a water invasion, we moved to second step to check the face-to-face neighbours of the voxels that had been invaded by water in the previous step; similarly, water invaded the neighbours whose capillary pressures were less than $p$. Applying and repeating these procedures to all pore voxels will find the pore voxels which would be invaded by water. If these pores hydraulically link the CL side and the GDL side, the water breaks the MPL. Using this method, we calculated the water-entry pressure, and the dependence of MPL saturation on the pressure built up by water in the CL. In all simulations, the four vertical sides of the image shown in Figure 3A were treated as periodic boundary.

\subsection{Oxygen diffusion and effective diffusion coefficient}

We focused on steady state and assumed that the water-air interfaces calculated in previous section were stagnant and did not change with time. Oxygen diffusion was restricted to the air phase. As shown in Figure 3B, the average pore sizes in the image were in the order of $10^{2} \mathrm{~nm}$. When oxygen moves in such pores, its collision with solid walls could be significant. The relative significance of oxygen-wall collision in a pore is described by the Knudsen number $\lambda / \delta$, where $\lambda$ is the average distance that an oxygen molecule travels between two consecutive collisions with other gaseous molecules, and $\delta$ is the size of the pore. Under the influence of both bulk and Knudsen 
resistances, the oxygen diffusion in a single pore can be described by the following modified Fick's law:

$$
\left(r_{B}+r_{K u}\right) \boldsymbol{q}=-\nabla c
$$

where $\boldsymbol{q}$ is the diffusive flux, $c$ is concentration, $r_{B}=D_{B}^{-1}$ and $r_{K u}=D_{K u}^{-1}$ with $D_{B}$ and $D_{K u}$ being the bulk and Knudsen diffusion coefficients, respectively, in the pore. Since the Knudsen diffusion coefficient depends on pore size, it varies spatially. Taking this into account, the oxygen diffusion through the air phase after water invasion into the image was described by

$$
\frac{\partial c}{\partial t}=-\nabla \cdot \boldsymbol{q}
$$

Following the work of Becker and his colleagues [30, 31], we assumed that the Knudsen diffusion coefficient of the oxygen in a pore is related to the pore diameter and the mean thermal velocity $\left(v_{\text {therm }}\right)$ of the oxygen as follows:

$D_{K u}=\frac{1}{3} \delta \cdot v_{\text {thermal }}$

The thermal velocity depends on temperature and molecular mass, and is given by [30]

$$
v_{\text {thermal }}=\sqrt{\frac{8 k T}{\pi m}},
$$

where $T$ is temperature, $k$ is the Boltzmamn number and $m$ is the molecular mass of the oxygen. At temperature of $45^{\circ} \mathrm{c}$, the thermal velocity is $v_{\text {thermal }}=58800 \mathrm{~cm} / \mathrm{s}$.

Since each pore voxel was associated with a pore and each pore has a diameter, each pore voxel in the image shown in Figure 3 has an associated Knudsen diffusion coefficient and its value was calculated by Eq. (3). The bulk diffusion coefficient is the same for all pores, and at temperature of $45^{\circ} \mathrm{C}$, its value is $D_{B}=0.24 \mathrm{~cm}^{2} / \mathrm{s}$.

Oxygen diffusion in the image was simulated under different saturations using a pore-scale model we developed for modelling oxygen diffusion and reaction in the catalyst layer [21]. In all 
simulations, oxygen diffusion was driven by a concentration gradient imposed in the GDL-CL direction, and the other four vertical sides were treated as periodic boundary. Inside the image, the air-solid and air-water interfaces were assumed to be impermeable and were solved as a zero-flux boundary. In each simulation, the oxygen concentration and flux at all pore voxels were sampled after the diffusion was deemed to have reached steady state. They were then spatially averaged to calculate the volumetric diffusive flux and concentration. The average concentration gradient and the diffusive flux were related through the following Fick's law:

$$
Q=-D_{e f f} \nabla C,
$$

where $Q$ is the average diffusive flux, $D_{\text {eff }}$ is the effective diffusion coefficient and $C$ is the average concentration. We used Eq. (5) to estimate the effective diffusion coefficient. For pore-scale simulation conducted at saturation of $\Theta$, the effective diffusion coefficient was calculated from

$$
D_{\text {eff }}(\Theta)=\frac{L_{x} \sum_{i=1}^{N} q_{i}^{x}}{N\left(c_{\text {int }}-c_{\text {out }}\right)}
$$

where $q_{i}^{x}$ is the diffusive flux in the GDL-CL direction at the ith voxel; $N$ is the number of air-filled

voxels; $L_{x}$, in terms of voxels, is the size of the image in the GDL-CL direction; $c_{\text {int }}$ and $c_{\text {out }}$ are the constant concentrations imposed at the inlet and outlet boundaries, respectively.

\section{Results analysis and discussion}

\subsection{Pore-size distribution and water invasion}

Figure 3B shows the pore-size distribution estimated for the image shown in Figure 3A. It is slightly skewed forwards, consisting of a few large pores with diameters of approximately 325 nanometres. Majority of the pores, however, have diameters in the region of 75 nanometres to 200 nanometres, consistent with the results found by other researchers [30]. The average pore-diameter is 141 nanometres. 
For easy analysis, we normalised the pressure $p$ built by water in the CL as $p^{\prime}=p \varepsilon /(\sigma \cdot \cos \theta)$, where $\varepsilon$ is the side-length of the voxels in the image. Figure 4A shows the liquid water clusters formed in the MPL when the normalised pressure is $p^{\prime}=0.125$; the associated saturation is $54 \%$. Figure $4 \mathrm{~B}$ shows the water distribution in the image, and Figure $4 \mathrm{C}$ is a $2 \mathrm{D}$ cross section. It is evident from Figure 4C that the water indeed invaded the big pores preferentially, leaving small pores filled by air. The two air-filled larger pores in Figure 4C are hydraulically isolated by small pores.

Figure 5A shows the dependence of the saturation on the normalised pressure. The normalised water-entry pressure is $p^{\prime}=0.09$, and the associated saturation with this pressure is $5 \%$. Such a small saturation, however, might not be sufficient to sustain drainage of liquid water from the CL to the GDL. If we assumed $30 \%$ is a reasonable saturation for the MPL to sustain water conduction, the associated normalised pressure that the water needs to build up in the CL would be 0.12 . At temperate of $45^{\circ} \mathrm{C}$, the water-air surface tension is $0.00007 \mathrm{kN} / \mathrm{m}$. The side-length of the voxels in the image is $1.4 \times 10^{-8} \mathrm{~m}$. In physical unit, the pressure that the water needs to build up in the CL so as to sustain water conduction increases with its hydrophobicity; the result is shown in Figure 5B.

The figure reveals that if the MPL is too hydrophobic, it would be physically impossible for the water to break through it. This is consistent with the observation of Aoyama et al. [24], where the liquid water accumulated in the CL but was unable to move into the MPL. Therefore, there must be other pathways for the liquid water to exit. One possibility is cracks that might have developed in their MPL [33-36]. Although Aoyama et al.[24] hypothesized that the water could have entered the MPL as water vapour, there must also be an exit for the liquid water condensed in the CL.

Development of cracks appears to be a common phenomenon in MPL [2, 33]. For example, Martinez-Rodriguez et al [37] found that the aperture of the cracks developed in their MPL was in the range of 4.4 microns to 32 microns. Furthermore, the density and aperture of the cracks developed in the MPL appear to increase with its hydrophobicity [38]. To elucidate the role of the 
cracks in water conduction, we take a MPL with moderate hydrophobicity in that its water contact angle is $110^{\circ}$ as an example. If cracks develop in such a MPL and their apertures are in the range of 4.4 microns to 32 microns, the capillary pressure of these cracks will be in the range of $1.5 \mathrm{kPa}$ to $10 \mathrm{kPa}$. These are lower than the pressure in a working cell, and are much easier for liquid water to move through, consistent with the experiments $[33,34]$. Even for the super-hydrophobic MPL with a water contact angle of $150^{\circ}$ [39], if similar cracks develop within it, their capillary pressure is also much lower than the pressure of an working cell.

The cracks appear be to be beneficial to water management without scarifying oxygen diffusion, but they will deteriorate the mechanical durability of the fuel cell assembly. There is no consensus in the literature over how hydrophobic a MPL should be, with some developing less hydrophobic MPL [40, 41], whilst others preferring high hydrophobic MPL[39]. A key parameter in understanding the role of MPL and to design it is to understand how its saturation will affect oxygen diffusion and water movement.

\subsection{Effective diffusion coefficient}

For easy analysis, in what follows we normalised all effective diffusion coefficients by the bulk diffusion coefficient in open air. For testing the dusty model, we separately calculated the effective bulk diffusion coefficient from pore-scale simulations by assuming the Knudsen diffusion in each pore was infinite, and the effective Knudsen diffusion coefficient from spore-scale simulations by assuming the bulk diffusion coefficient in each pore was infinite. Figure 6 shows the change of the two effective diffusion coefficients with saturation. Although the effective Knudsen diffusion coefficient is lightly smaller than the bulk one, they are comparable, revealing the significance of the oxygen-wall collisions. Both effective diffusion coefficients decrease with saturation $S$, and can be fitted to $D_{\text {eff }} / D_{B}=A(1-S)^{3.1}$ with $A=0.24$ for the effective bulk diffusion coefficient and $A=0.19$ for the effective Knudsen diffusion coefficient. 
In a single pore, the bulk and Knudsen diffusion coefficients can be approximated by a combined diffusion coefficient as $D=\left(D_{B}^{-1}+D_{K u}^{-1}\right)^{-1}$ to describe oxygen diffusion in the single pore. In some literature, this approximation was extrapolated to calculate the effective diffusion coefficient at macroscopic scale, i.e., $D_{\text {eff }}=\left(D_{\text {Beff }}^{-1}+D_{\text {Kueff }}^{-1}\right)^{-1}$ in which $D_{\text {Beff }}$ and $D_{\text {Kueff }}$ are the effective bulk and Knudsen diffusion coefficients respectively [31]. Physically, there is an interaction between the bulk and the Knudsen diffusions in each single pore, and the above approximation assumes that this interaction can be averaged out after the volumetric average. To test its reliability, we compared the effective diffusion coefficient estimated from this approximation with those directly calculated from pore-scale simulations based on Eq. (2) using a combined diffusion coefficient for each pore voxel. Figure 7 shows the comparison; there is a noticeable difference between them, and the diffusion coefficient estimated by the above approximation is nearly three times higher than those directly calculated from the pore-scale simulations. This implies that the interaction between the bulk and Knudsen diffusions at pore scale cannot be averaged out, and instead, they create an extra resistance to oxygen diffusion after the volumetric average. It is well known in other areas that spatial average of any processes will introduce an extra resistance. For example, in fluid mechanics, the volumetric average of a turbulent flow creates a turbulent-viscosity, which is much larger than fluid's dynamics viscosity. It is anticipated that similar mechanisms also happen to oxygen diffusion in nanopores in that the volumetric average leads to an extra resistance.

The effective diffusion coefficient directly calculated from the pore-scale simulation decreases with saturation, and the decrease can be fitted to $D_{\text {eff }} / D_{B}=A(1-S)^{3.1}$ with $A=0.038$. The accuracy of this fitting is shown in Figure 7.

\section{Conclusions}


Hydrophobic MPL had been proven capable of improving water management in PEM fuel cells, but the underlying mechanisms are not fully understood. The MPL affects cell performance because it changes the processes of water flow and oxygen diffusion, and understanding this change is important in MPL design. In this paper, we investigated these processes using tomography and pore-scale modelling. Image of a MPL was acquired using focused ion beam/scanning electron microscopy (FIB/SEM) at resolution of 14 nanometres; pore-scale models were developed to simulate water flow and oxygen diffusion in the image under different saturations. In the simulations, the water was assumed to condense in the CL. Since the MPL is more hydrophobic, the water needs to build up a pressure first before moving into the MPL. Based on the pore sizes and pore connectedness in the MPL, we calculated the relationship between its saturation and the pressure that the water built up in the CL. We also calculated the minimum pressure that the water is required to build up in the CL in order to move through the MPL, and linked this pressure to MPL hydrophobicity. The result indicates that, for highly hydrophobic MPL, it is physically impossible for the liquid water to flow through it, and the water has to find alternative exits, such as cracks developed in MPL. However, MPL with cracks has detrimental effect on its mechanical durability. As such, crack-less MPL is preferred, for which we need to understand the impact of its saturation on water flow and oxygen diffusion.

Pore-scale oxygen diffusions in the image under different saturations were also simulated. We considered both bulk and Knudsen diffusions, and imposed a concentration gradient in the GDL-CL direction to drive the oxygen. The simulated concentration and flux at all voxels were volumetrically averaged, and the results were used to calculate the effective diffusion coefficient. To test the dusty model, we calculated three effective diffusion coefficients: effective bulk diffusion coefficient calculated assuming the Knudsen diffusion was infinite, effective Knudsen diffusion coefficient calculated assuming the bulk diffusion was infinite, and effective diffusion coefficient calculated by combining the bulk and Knudsen diffusion coefficients in each single pore. 
The results show that all the three effective diffusion coefficients decrease with saturation, and the decrease can be described by $D_{\text {eff }} / D_{B}=A(1-S)^{3.1}$ with $A=0.24$ for the effective bulk diffusion coefficient, $A=0.201$ for the bulk Knudsen diffusion coefficient and $A=0.0385$ for the effective diffusion coefficient considering both Knudsen and bulk diffusions.

The dusty model was derived under an assumption that the interplay of the bulk and the Knudsen diffusions at pore scale could be averaged out after the volumetric average. Our results show that this approximation substantially overestimated the effective diffusion coefficient, implying that the volumetric average leads to an extra resistance. This is a phenomenon similar to turbulence flow where a spatial average results in a turbulent viscosity.

There has been an increased interest in use of MPL to improve water management. While highly hydrophobic MPLs appear to work well in improving water management without scarifying oxygen diffusion, they must have developed cracks. Depending on their apertures and lengths, these cracks could have a detrimental impact on mechanical durability of the cell assembly. In the literature, there have been efforts in developing both less-hydrophobic and super-hydrophobic MPL[39, 40]. To understand the roles of different MPLs, one needs to understand the impact of their saturation on water flow and oxygen diffusion first. Since the MPL is only a few microns thick, experimentally measuring these parameters and testing their impact on cell performance is difficult. Pore-scale modelling and tomography provide an alternative to bridge this gap, and this paper makes such an attempt. Our results elucidated some mechanisms involved in water movement in MPL, and showed how this would affect the oxygen diffusion when the Knudsen diffusion becomes significant.

\section{Acknowledgements}

Part of this research was supported by the UK Technology Strategy Board (TSB Project No. $\mathrm{TP} / 6 / \mathrm{S} / \mathrm{K} 3032 \mathrm{H})$. 


\section{References}

[1] Dai W, Wang HJ, Yuan XZ, Martin JJ, Yang DJ, Qiao JL, et al. A review on water balance in the membrane electrode assembly of proton exchange membrane fuel cells. Int J Hydrog Energy. 2009;34:9461-78.

[2] Wang XL, Zhang HM, Zhang JL, Xu HF, Tian ZQ, Chen J, et al. Micro-porous layer with composite carbon black for PEM fuel cells. Electrochim Acta. 2006;51:4909-15.

[3] Qi ZG, Kaufman A. Improvement of water management by a microporous sublayer for PEM fuel cells. J Power Sources. 2002;109:38-46.

[4] Ramasamy RP, Kumbur EC, Mench MM, Liu W, Moore D, Murthy M. Investigation of macroand micro-porous layer interaction in polymer electrolyte fuel cells. Int J Hydrog Energy.

2008;33:3351-67.

[5] Gostick JT, Ioannidis MA, Fowler MW, Pritzker MD. On the role of the microporous layer in PEMFC operation. Electrochem Commun. 2009;11:576-9.

[6] Weng FB, Hsu CY, Su MC. Experimental study of micro-porous layers for PEMFC with gradient hydrophobicity under various humidity conditions. Int J Hydrog Energy. 2011;36:1370814.

[7] Schweiss R, Steeb M, Wilde PM. Mitigation of Water Management in PEM Fuel Cell Cathodes by Hydrophilic Wicking Microporous Layers. Fuel Cells. 2010;10:1176-80.

[8] Lobato J, Canizares P, Rodrigo MA, Ubeda D, Pinar FJ, Linares JJ. Optimisation of the Microporous Layer for a Polybenzimidazole-Based High Temperature PEMFC - Effect of Carbon Content. Fuel Cells. 2010;10:770-7.

[9] Chan C, Zamel N, Li XG, Shen J. Experimental measurement of effective diffusion coefficient of gas diffusion layer/microporous layer in PEM fuel cells. Electrochim Acta. 2012;65:13-21.

[10] Mukherjee PP, Kang QJ, Wang CY. Pore-scale modeling of two-phase transport in polymer electrolyte fuel cells-progress and perspective. Energy Environ Sci. 2011;4:346-69.

[11] Cecen A, Wargo EA, Hanna AC, Turner DM, Kalidindi SR, Kumbur EC. 3-D Microstructure Analysis of Fuel Cell Materials: Spatial Distributions of Tortuosity, Void Size and Diffusivity. J Electrochem Soc. 2012;159:B299-B307.

[12] Kim SG, Lee SJ. Tomographic analysis of porosity variation in gas diffusion layer under freeze-thaw cycles. Int J Hydrog Energy. 2012;37:566-74.

[13] Ostadi H, Rama P, Liu Y, Chen R, Zhang X, Jiang K. Nanotomography based study of gas diffusion layers. Microelectron Eng. 2010;87:1640-2.

[14] Ostadi H, Rama P, Liu Y, Chen R, Zhang XX, Jiang K. 3D reconstruction of a gas diffusion layer and a microporous layer. J Membr Sci. 2010;351:69-74.

[15] Thiele S, Zengerle R, Ziegler C. Nano-morphology of a polymer electrolyte fuel cell catalyst layer-imaging, reconstruction and analysis. Nano Research. 2011;4:849-60.

[16] Ziegler C, Thiele S, Zengerle R. Direct three-dimensional reconstruction of a nanoporous catalyst layer for a polymer electrolyte fuel cell. Journal of Power Sources. 2011;196:2094-7.

[17] Wargo EA, Schulz VP, Cecen A, Kalidindi SR, Kumbur EC. Resolving macro- and microporous layer interaction in polymer electrolyte fuel cells using focused ion beam and X-ray computed tomography. Electrochim Acta. 2013;87:201-12.

[18] Hao L, Cheng P. Lattice Boltzmann simulations of water transport in gas diffusion layer of a polymer electrolyte membrane fuel cell. Journal of Power Sources. 2010;195:3870-81.

[19] Kamarajugadda S, Mazumder S. Numerical investigation of the effect of cathode catalyst layer structure and composition on polymer electrolyte membrane fuel cell performance. Journal of Power Sources. 2008;183:629-42.

[20] Wargo EA, Kotaka T, Tabuchi Y, Kumbur EC. Comparison of focused ion beam versus nanoscale X-ray computed tomography for resolving 3-D microstructures of porous fuel cell materials. Journal of Power Sources. 2013;241:608-18. 
[21] Zhang X, Ostadi H, Jiang K, Chen R. Reliability of the spherical agglomerate models for catalyst layer in polymer electrolyte membrane fuel cells. Electrochim Acta. 2014;133:475-83. [22] Li H, Tang YH, Wang ZW, Shi Z, Wu SH, Song DT, et al. A review of water flooding issues in the proton exchange membrane fuel cell. Journal of Power Sources. 2008;178:103-17.

[23] Pasaogullari U, Wang CY, Chen KS. Two-phase transport in polymer electrolyte fuel cells with bilayer cathode gas diffusion media. J Electrochem Soc. 2005;152:A1574-A82.

[24] Aoyama Y, Suzuki K, Tabe Y, Chikahisa T. Observation of water transport in the microporous layer of a polymer electrolyte fuel cell with a freezing method. and cryo-scanning electron microscope. Electrochem Commun. 2014;41:72-5.

[25] Nanjundappa A, Alavijeh AS, El Hannach M, Harvey D, Kjeang E. A customized framework for 3-D morphological characterization of microporous layers. Electrochim Acta. 2013;110:349-57. [26] Fishman Z, Bazylak A. Heterogeneous Through-Plane Porosity Distributions for Treated PEMFC GDLs. II. Effect of MPL Cracks. J Electrochem Soc. 2011;158:B846-B51.

[27] Kalidindi AR, Taspinar R, Litster S, Kumbur EC. A two-phase model for studying the role of microporous layer and catalyst layer interface on polymer electrolyte fuel cell performance. Int $\mathbf{J}$ Hydrog Energy. 2013;38:9297-309.

[28] Kandlikar SG, See EJ, Koz M, Gopalan P, Banerjee R. Two-phase flow in GDL and reactant channels of a proton exchange membrane fuel cell. Int J Hydrog Energy. 2014;39:6620-36.

[29] Pant LM, Mitra SK, Secanell M. Absolute permeability and Knudsen diffusivity measurements in PEMFC gas diffusion layers and micro porous layers. Journal of Power Sources. 2012;206:153-

60.

[30] Becker J, Wieser C, Fell S, Steiner K. A multi-scale approach to material modeling of fuel cell diffusion media. Int J Heat Mass Transf. 2011;54:1360-8.

[31] Hutzenlaub T, Becker J, Zengerle R, Thiele S. Modelling the water distribution within a hydrophilic and hydrophobic 3D reconstructed cathode catalyst layer of a proton exchange membrane fuel cell. Journal of Power Sources. 2013;227:260-6.

[32] Wargo EA, Hanna AC, Cecen A, Kalidindi SR, Kumbur EC. Selection of representative volume elements for pore-scale analysis of transport in fuel cell materials. Journal of Power Sources. 2012;197:168-79.

[33] Sasabe T, Deevanhxay P, Tsushima S, Hirai S. Soft X-ray visualization of the liquid water transport within the cracks of micro porous layer in PEMFC. Electrochem Commun. 2011;13:63841.

[34] Markotter H, Haussmann J, Alink R, Totzke C, Arlt T, Klages M, et al. Influence of cracks in the microporous layer on the water distribution in a PEM fuel cell investigated by synchrotron radiography. Electrochem Commun. 2013;34:22-4.

[35] Deevanhxay P, Sasabe T, Tsushima S, Hirai S. Effect of liquid water distribution in gas diffusion media with and without microporous layer on PEM fuel cell performance. Electrochem Commun. 2013;34:239-41.

[36] Deevanhxay P, Sasabe T, Tsushima S, Hirai S. Observation of dynamic liquid water transport in the microporous layer and gas diffusion layer of an operating PEM fuel cell by high-resolution soft X-ray radiography. Journal of Power Sources. 2013;230:38-43.

[37] Martinez-Rodriguez MJ, Cui T, Shimpalee S, Seraphin S, Duong B, Van Zee JW. Effect of microporous layer on MacMullin number of carbon paper gas diffusion layer. J Power Sources. 2012;207:91-100.

[38] Ahn M, Cho YH, Kim J, Jung N, Sung YE. Influence of hydrophilicity in micro-porous layer for polymer electrolyte membrane fuel cells. Electrochim Acta. 2011;56:2450-7.

[39] Latorrata S, Stampino PG, Cristiani C, Dotelli G. Novel superhydrophobic microporous layers for enhanced performance and efficient water management in PEM fuel cells. Int J Hydrog Energy. 2014;39:5350-7. 
[40] Chun JH, Park KT, Jo DH, Lee JY, Kim SG, Park SH, et al. Development of a novel hydrophobic/hydrophilic double micro porous layer for use in a cathode gas diffusion layer in PEMFC. Int J Hydrog Energy. 2011;36:8422-8.

[41] Chun JH, Jo DH, Kim SG, Park SH, Lee CH, Kim SH. Improvement of the mechanical durability of micro porous layer in a proton exchange membrane fuel cell by elimination of surface cracks. Renew Energy. 2012;48:35-41. 


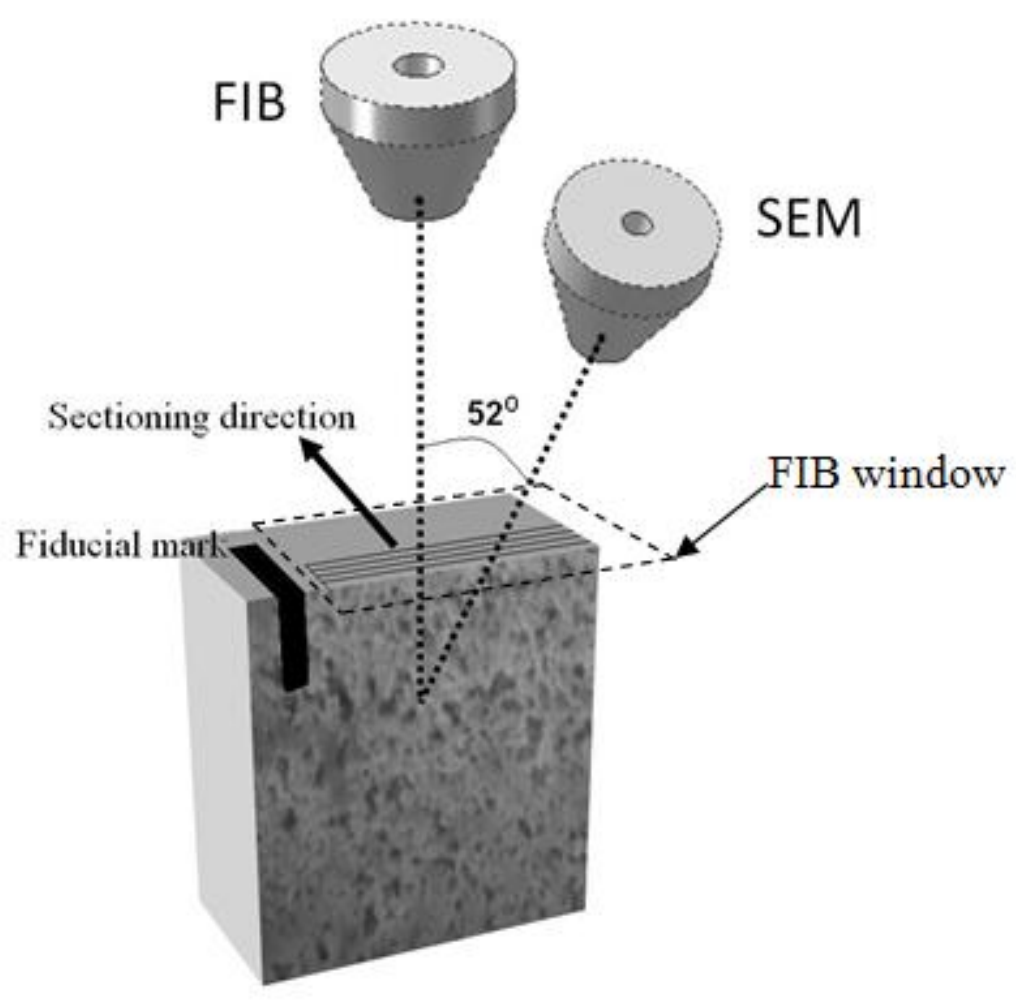

Figure 1. The configuration of FIB/SEM nanotomography. A cubic fiducial mark is milled first. Then the sample is milled in $10 \mathrm{~nm}$ thickness before an SEM image of the sample is taken. This milling-imaging cycle is repeated and over a hundred of SEM images are collected. The sectioning direction was perpendicular to FIB column. The images were aligned in reference to the fiducial mark and assembled into a 3D object. FIB window is shown in dashed line. With this configuration the small fiducial mark is protected against FIB bombardment. 


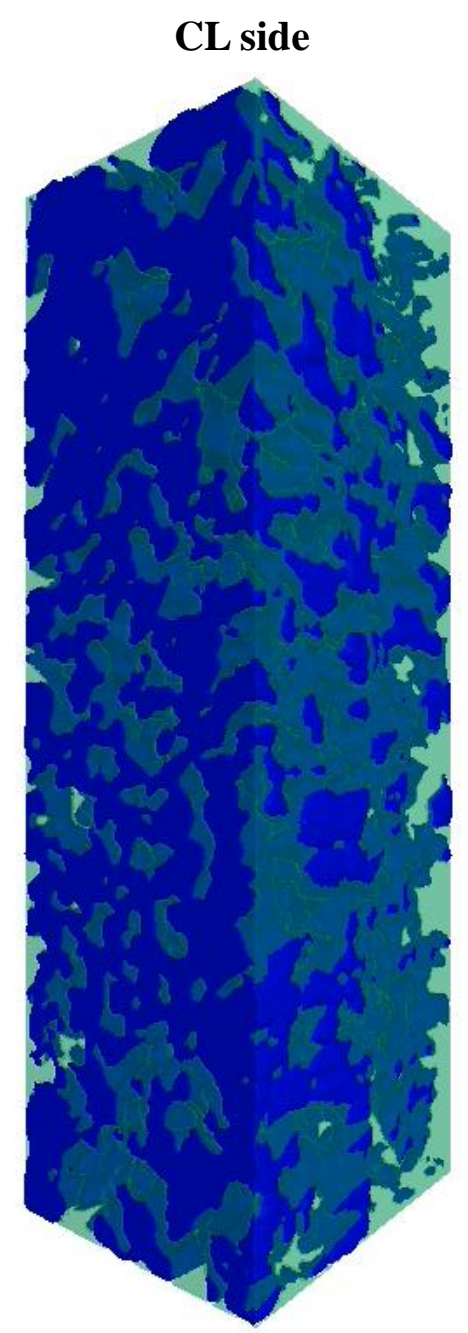

GDL side

Figure 2. The reconstructed binary image of the MPL acquired using FIB/SEM at resolution of 14 nanometres (the blue is solid and the grey is pores). 


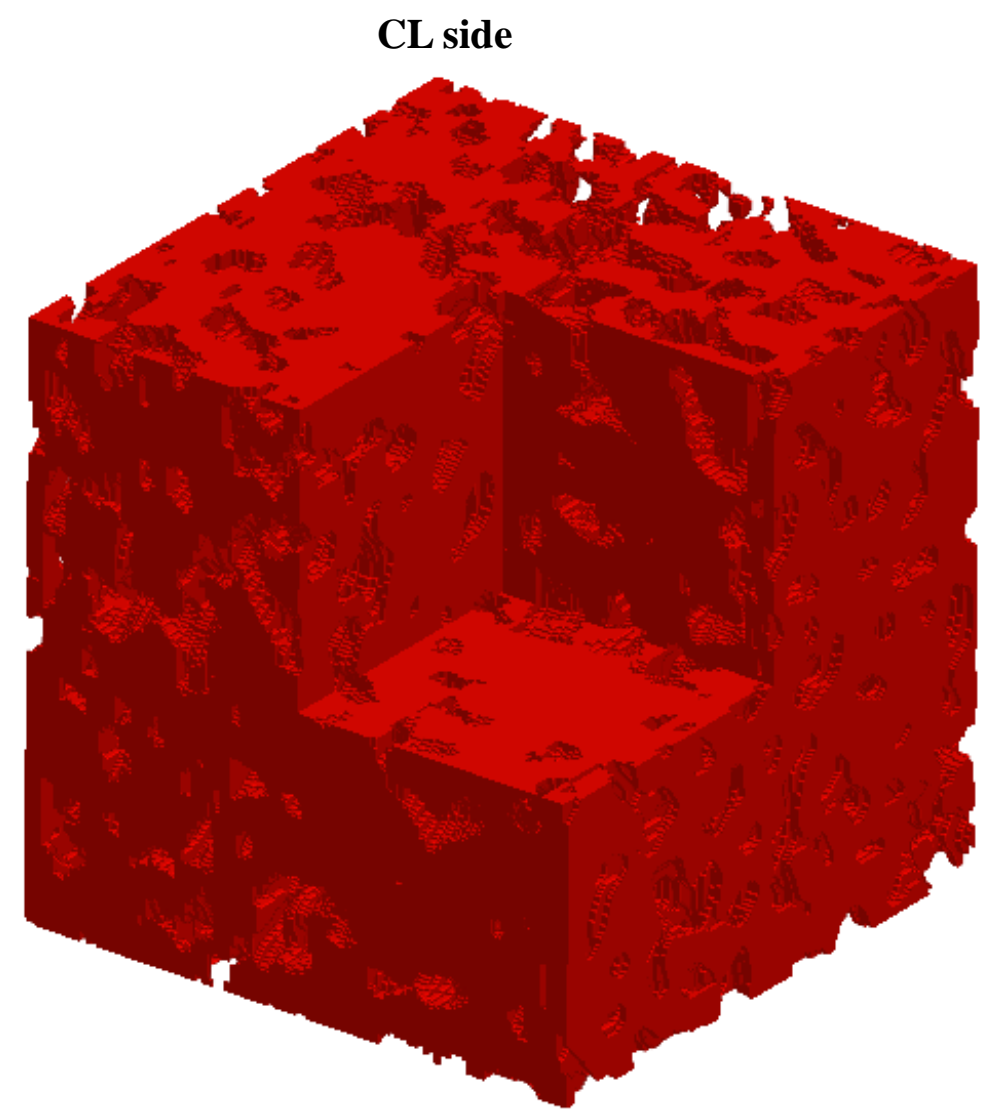

GDL side

(A)

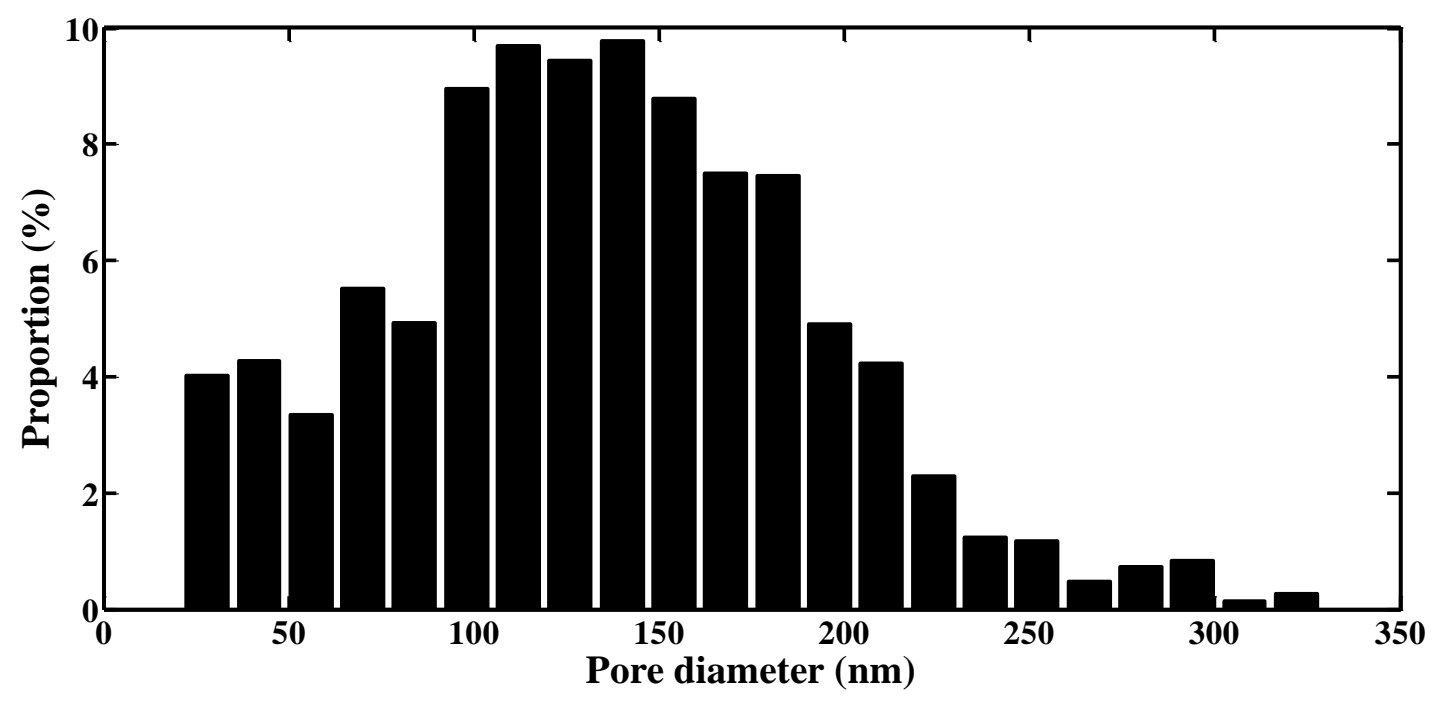

(B)

Figure 3. (A) The MPL image used in the simulations; (B) the pore-size distribution estimated from the $3 \mathrm{D}$ image. 


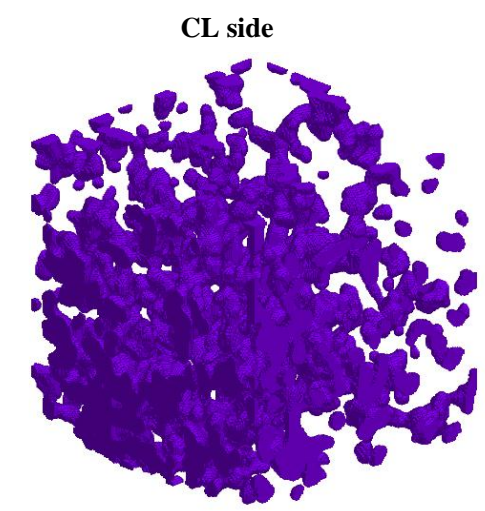

GDL side

(A)

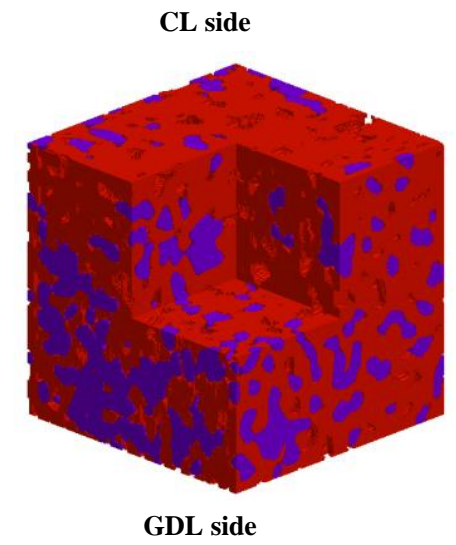

(B)

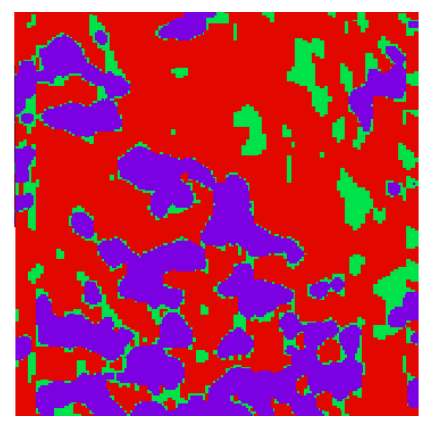

(C)

Figure 4. An illustration of water invasion and distribution in the MPL under a normalised waterentry pressure of 0.15 ; the associated saturation is 0.54 . (A) The clusters formed by the invading water in the MPL(the air and solid are made transparent); (B) water distribution in the MPL (the air is made transparent); (C) a horizontal 2D slice showing the preferential invasion of water into the big pores. 


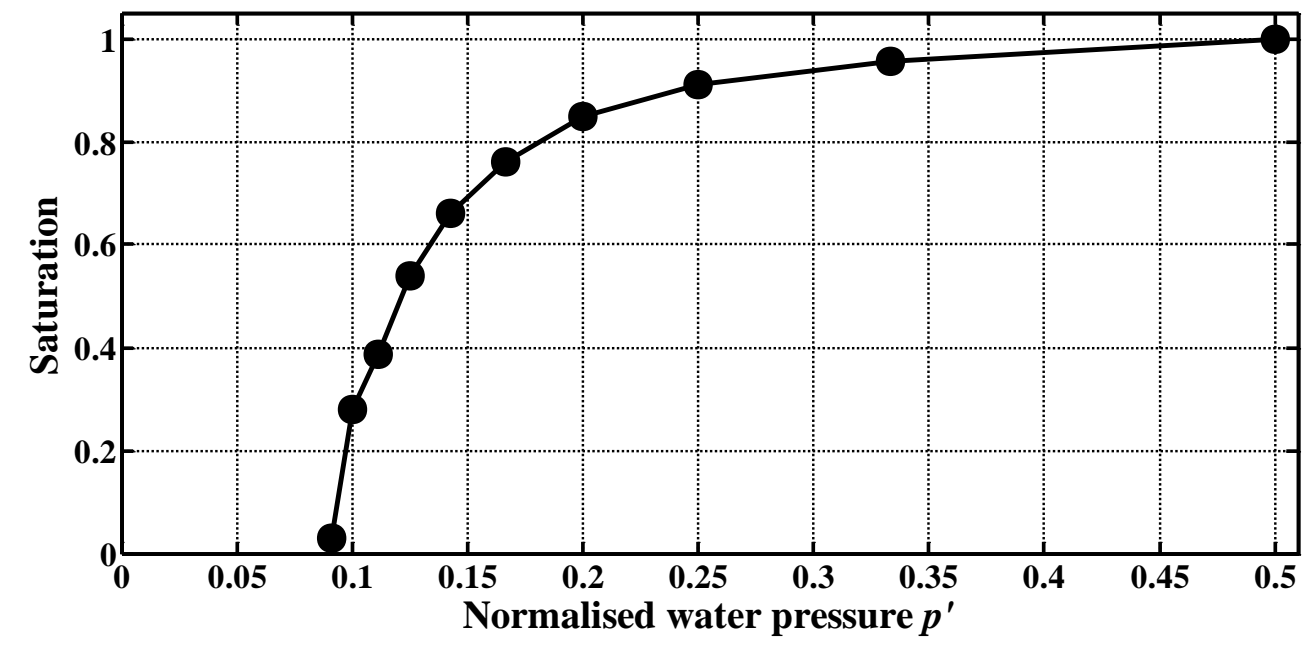

(A)

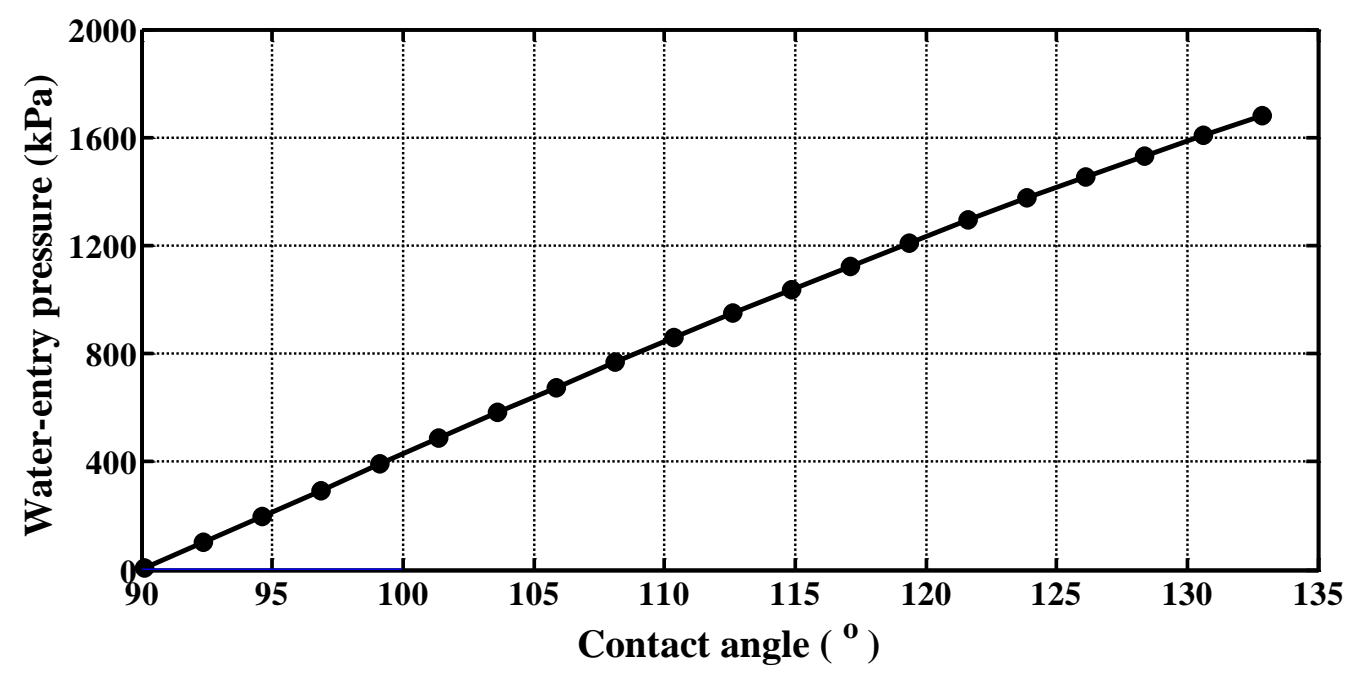

(B)

Figure 5. (A) The relationship between saturation of the MPL and the normalised pressure built by water in the CL. (B) Change of the water-entry pressure with water contact angle when keeping the saturation of the MPL at $30 \%$. 


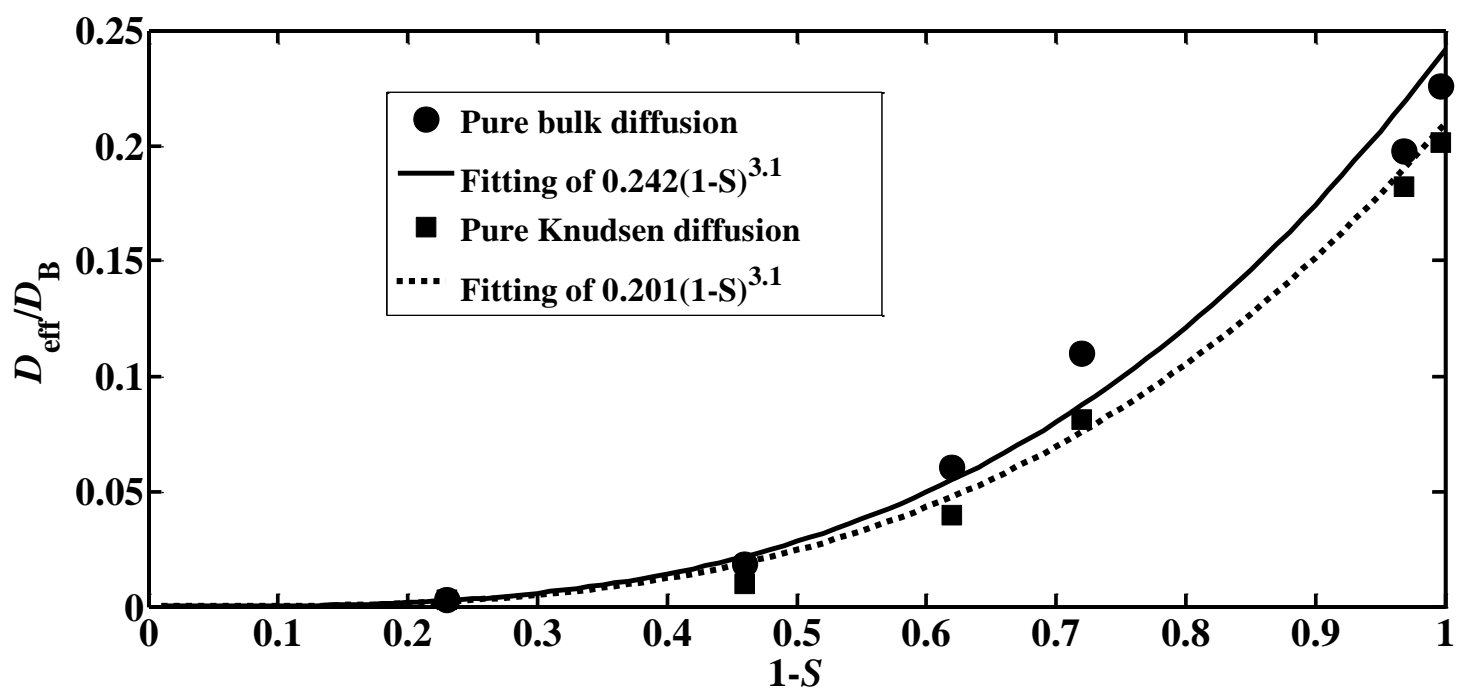

Figure 6. Change of the calculated effective bulk and Knudsen diffusion coefficients with saturation. 


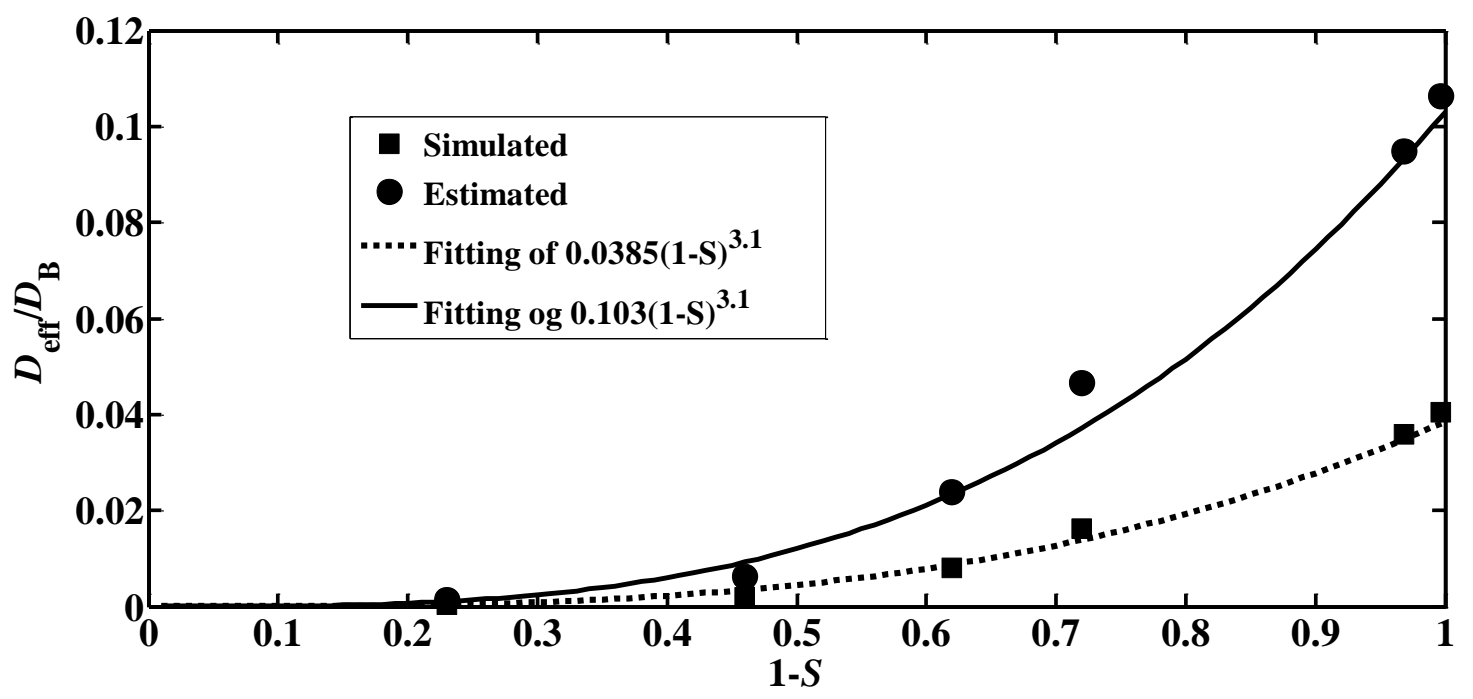

Figure 7. Comparison between the effective diffusion coefficients directly calculated from porescale simulations with that estimated from the dusty model using the effective bulk and Knudsen diffusion coefficients shown in Figure 6 under different saturations. 